\title{
The Two-Point Correlation Function of Ringed Galaxies
}

\author{
Wentao Wu, Renato A. Dupke and Carlos R. Rabaça \\ Department of Physics $\mathcal{B}$ Astronomy, The University of Alabama, Box \\ 870324, Tuscaloosa, AL 35487
}

\begin{abstract}
We calculate the two-point correlation function for a sample of ringed galaxies. Results are similar to that of general field galaxies. We also find evidence that these galaxies form very large scale structures.
\end{abstract}

\section{Introduction}

The analysis of the large scale structure of the universe provides constraints on cosmological models. The two-point correlation function (TPCF) is the minimum constraint on these models. TPCFs for several different populations have been determined and show the existence of a universal dimensionless correlation (Bahcall \& West 1992). We apply the TPCF to a sample of ringed galaxies to obtain the cosmological implication of this population of galaxies.

\section{Sample and Calculations}

Our sample contains 359 inner and 264 outer ringed galaxies from the Catalog of Southern Ringed Galaxies (CSRG, Buta 1995) with: (1) $\delta(1950)<-42^{\circ}$; (2) $b_{I I} \leq-19.0^{\circ}$ (outer) and $-15.5^{\circ}$ (inner ringed galaxies); and (3) apparent ring major axis $\geq 1.0^{\prime}$ (outer) and $0.5^{\prime}$ (inner ring). No significant Galactic latitude selection effect was found in this sample from the examination of the galaxy surface number density distribution with Galactic latitude. The mean surface densities are $\sigma=222$ and $277 \mathrm{rad}^{-2}$ for outer and inner ringed galaxies, respectively.

We used two methods to calculate the TPCF, $w(\theta)$. The standard method (SM, Peebles 1980) is based on the homogeneity assumption, i.e., the distributions of galaxies or galaxy associations inside and outside the boundaries of the sample are the same. It works well for small angular scales, but for large angular scales the homogeneity assumption may be compromised. The alternative method used was the reference subsample method (RSM, Wiedenmann \& Atmanspacher 1990). The fair reference subsample assumption consists of averaging the correlations for galaxies in small enough subsamples, thus avoiding the boundary problem. The TPCFs (Figure 1) were calculated by comparing the number of pairs in the real samples with those in artificially generated homogeneous samples. The power-law $w(\theta)=\left(\theta / \theta_{0}\right)^{-\beta}$ fits the data well in scales up to $10^{\circ}$. The slopes found are very close (within the errors) to the one for field galaxies ( $\beta \sim 0.8$, Peebles 1993). 


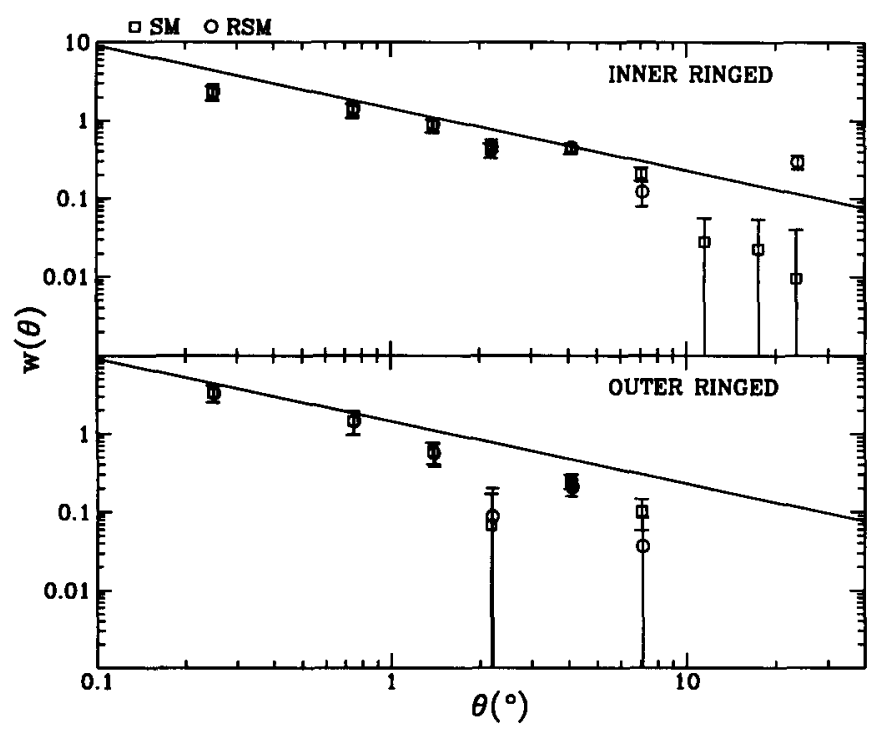

Figure 1. TPCF for ringed galaxies. Solid lines show the slope for field galaxies, for comparison.

We also calculated the integral TPCF

$$
\gamma(\theta)=\int_{0}^{\theta}\left(1+w\left(\theta^{\prime}\right)\right) d\left(1-\cos \theta^{\prime}\right) /(1-\cos \theta) .
$$

It gives the probability of finding a galaxy within a separation $\theta$ from any galaxy relative to a uniform distribution. If $\gamma(\theta) \rightarrow 1$ the whole distribution approaches homogeneity. By examining the behavior of $\gamma(\theta)$ we find structures at least up to $10^{\circ}\left(\gamma\left(\leq 10^{\circ}\right)>1\right)$.

\section{Discussion}

We found that the slopes of the TPCF of ringed galaxies are similar to that of field galaxies, suggesting that the processes of formation of large scale structures are similar to both populations.

It is a good approximation to assume a constant major axis for outer/inner rings, which is is supported by the number distribution of apparent ring major axis (Buta 1995). We estimated from a few dozens of NGC and IC galaxies with known redshifts in the CSRG that the typical physical major axes are 10 and 20 $h^{-1} \mathrm{kpc}$ for inner and outer rings, respectively. These considerations together with the sample limits on apparent ring sizes give an approximate effective depth of the sample $D_{*} \approx 70 h^{-1}$ Mpc. From Limber's equation (Peebles 1993) and taking a redshift selection function $\Psi$ of the form

$$
\Psi\left(D^{2} / D_{*}^{2}\right)= \begin{cases}\text { constant }, & D<D_{*} \\ 0, & \left.D \geq D_{*}, \quad \text { for constant ring size }\right)\end{cases}
$$




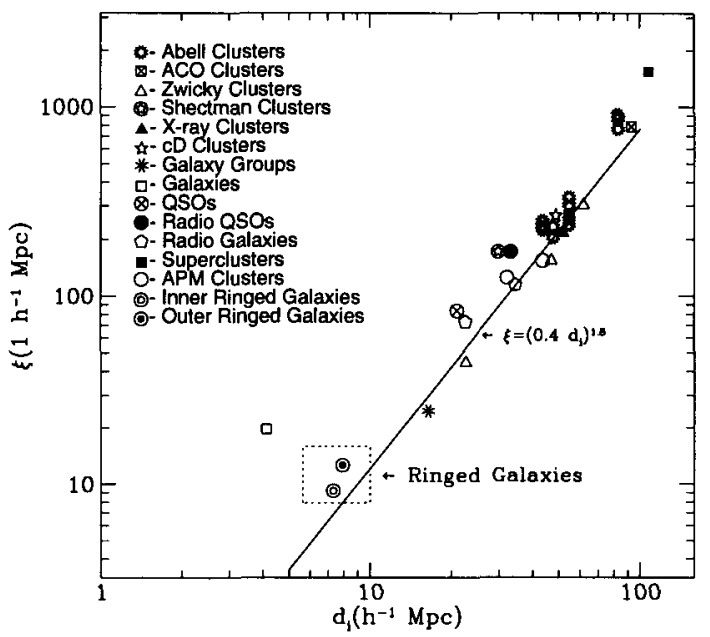

Figure 2. The universal correlation function.

the spatial correlation length for ringed galaxies is $r_{0} \approx 3.7-3.8 h^{-1} \mathrm{Mpc}$, close to the value for field galaxies, $\approx 5 h^{-1} \mathrm{Mpc}$. The mean separations $d_{i}=D_{*} /(3 \sigma)^{1 / 3} \approx 7.4$ and $8.0 h^{-1} \mathrm{Mpc}$, for outer and inner ringed galaxies, respectively. This shows (Figure 2) that the ringed galaxy TPCFs satisfy the universal dimensionless correlation function (Bahcall \& West 1992), $\xi_{1 \mathrm{Mpc}}=\left(r_{0} / 1 h^{-1} \mathrm{Mpc}\right)^{\nu} \approx\left(0.4 d_{i}(\mathrm{Mpc})\right)^{1.8}$. It should be noticed that this result is not sensitive to the specific value of $D_{*}$; since both $r_{0}$ and $d_{i}$ are scaled by $D_{*}$. This suggests that the "seeding" processes of ringed galaxies are similar to those of other known galaxy populations (QSOs, clusters, radio galaxies, etc).

The angular scale of the largest structures estimated from the integral TPCF $\left(\theta \approx 10-20^{\circ}\right)$ corresponds to a spatial scale $D_{*} \theta \approx 10-25 h^{-1} \mathrm{Mpc}$, comparable to sizes of superclusters or bubbles.

Acknowledgments. We thank R. Buta for providing the CSRG and the Alabama Space Grant Consortium for supporting our attendance of the meeting.

\section{References}

Bahcall, N. A. \& West, M. J. 1992, ApJ, 392, 419

Buta, R. 1995, ApJS, 96, 39

Peebles, P. J. E. 1980, The Large Scale Structure of the Universe, Princeton: Princeton University Press

Peebles, P. J. E. 1993, Principles of Physical Cosmology, Princeton: Princeton University Press

Wiedenmann, G. \& Atmanspacher, H. 1990, A\&A, 229, 283 